\title{
EDN3 Gene
}

National Cancer Institute

\section{Source}

National Cancer Institute. EDN3 Gene. NCI Thesaurus. Code C147998.

This gene is involved in vasoconstriction. 